\title{
Therapeutic Effect of Sodium Hyaluronate and Corticosteroid Injections on Pain and Temporomandibular Joint Dysfunction: A Quasi-experimental Study
}

\author{
Percy Romero-Tapia ${ }^{1}$, Fernando Pérez-Vargas ${ }^{2}$, Gabriela Sedano-Balbin ${ }^{3}$, Jorge Marín ${ }^{4}$, Frank Mayta-Tovalino ${ }^{5}$
}

\begin{abstract}
Aim: The aim was to evaluate and compare the therapeutic effect of sodium hyaluronate (SH) and corticosteroids (CS) on pain and temporomandibular dysfunction (TMD).

Materials and methods: Thirty patients with TMD [characterized by painful symptoms, affectations, and limitations of the mandibular movement and noises or clicks in the temporomandibular joint (TMJ)] were selected and divided into two equal groups nonrandomized. The clinical questionnaire was applied to each patient before performing the clinical procedures and included a visual analog scale with progressive values from 0 to 10 for the measurement of pain and clinical examination: maximum oral aperture (MOA), mandibular laterotrusive movement (MLM), and maximum protrusion (MP), before and after infiltration intra-articular with SH and CS up to 2 months.

Results: Both groups described benefits from treatment at the 1 st and 2 nd weeks, and at 1 and 2 months of follow-up, presenting a reduction in TMJ pain, and improvement in mandibular mobility with an increase of the MOA, lateralities, and protrusion. No statistically significant difference was found among these variables between the two drugs. However, SH showed a greater therapeutic effect in relation to a reduction in joint noises compared to CS.

Conclusion: Intra-articular infiltration with SH and CS in TMD is effective for the relief of TMJ pain and also reduces joint noises and improves mandibular mobility. Both drugs can be used with similar optimal results in the treatment of TMD.

Clinical significance: This research allows to know what is the therapeutic impact of injectable CS on the TMJ.

Keywords: Pain, Quasi-experimental study, Sodium hyaluronate, Temporomandibular joint dysfunction.

The Journal of Contemporary Dental Practice (2020): 10.5005/jp-journals-10024-2890
\end{abstract}

\section{INTRODUCTION}

The temporomandibular joint (TMJ), important structure of the stomatognathic system, is often compromised by inflammatory, infectious, traumatic, congenital, neoplastic, and developmental disorders. In addition, functional alterations can cause pain, with a greater predominance in women, and which is known as "syndrome of painful TMJ," "disorders of the TMJ," or simply "temporomandibular dysfunction (TMD)." Subjects with this disease generally refer to pain and have limitation of mandibular mobility, in addition to noise or clicks in the TMJ. ${ }^{1-4}$

Temporomandibular dysfunction, also known as temporomandibular disorder or craniomandibular dysfunction, is a group of abnormalities that affect the TMJ and the anatomical structures that surround it, sometimes constituting an unusual underdiagnosed cause of headache and myofacial painful disorders in a large percentage of the population. ${ }^{5-8}$

The dysfunctions that are normally established in the TMD include osteoarthritis, bone or cartilage pathology, mechanical disorder, inflammatory arthropathies, progressive ossifying myositis, etc. The mechanism of action of sodium hyaluronate ( $\mathrm{SH}$ ) is due to the fact that it is a high-molecular-weight polysaccharide composed of an extensive chain of disaccharides (B-D-glucoronylB-D-N-acetylglucosamine), fundamental in the composition of the LS and the articular disk and plays a primary role in the functioning of the synovial joints. Otherwise, the mechanism of action of corticosteroids (CS) is to participate in the inflammatory process by blocking the capillary permeability and reducing the exit of proteins and fluid into the extravascular space, reducing edema. ${ }^{2,5,7,9}$ \begin{tabular}{l}
\hline 1,4 Academic Department of Surgical Medical Stomatology, Faculty of \\
Dentistry, Universidad Nacional Mayor de San Marcos, Lima, Peru \\
${ }^{2}$ Department of Pediatric Stomatology, Faculty of Dentistry, \\
Universidad Nacional Mayor de San Marcos, Lima, Peru \\
${ }^{3}$ Department of Rehabilitative Stomatology, Faculty of Dentistry, \\
Universidad Nacional Mayor de San Marcos, Lima, Peru \\
${ }^{5}$ Postgraduate Department, Faculty of Health of Sciences, Universidad \\
Cientifica del Sur, Lima, Peru \\
Corresponding Author: Frank Mayta-Tovalino, Postgraduate \\
Department, Faculty of Health of Sciences, Universidad Cientifica del \\
Sur, Lima, Peru, Phone: +51 1 214-2500, e-mail: fmaytat@ucientifica. \\
edu.pe \\
How to cite this article: Romero-Tapia P, Pérez-Vargas F, Sedano-Balbin \\
G, et al. Therapeutic Effect of Sodium Hyaluronate and Corticosteroid \\
Injections on Pain and Temporomandibular Joint Dysfunction: A Quasi- \\
experimental Study. J Contemp Dent Pract 2020;21(10):1084-1090. \\
Source of support: Nil \\
Conflict of interest: None \\
\hline \hline
\end{tabular}

The conservative treatments of TMJ include rest, the use of nonsteroidal anti-inflammatory drugs (NSAIDs), CS, botulinum toxin injections, myo-relaxing splints, and physical therapy. Infiltration of drugs into the upper compartment of the TMJ can be performed associated or not with arthrocentesis, as an adjuvant method for the relief of symptoms and improvement of mandibular dynamics. ${ }^{3-5}$ Drugs usually used for infiltration are local anesthetics, NSAIDs, 
$\mathrm{CS}$, and $\mathrm{SH}$, among others. The use of intra-articular injections of high-molecular-weight SH achieves lubrication, viscous TMJ supplementation, and also provides a protective effect on the joint disk. This substance, which constitutes the majority of synovial fluid, is responsible for the lubrication of synovial joints, including TMJ. ${ }^{1,2,8,9}$

It has been shown that the individual use of $\mathrm{SH}$ and $\mathrm{CS}$ as exogenous viscous supplements in TMD is effective in the treatment of joint disorders. ${ }^{2,3,5,9}$ However, despite this, there are not many studies comparing these interventions simultaneously in TMD. ${ }^{9,10}$ Therefore, this study aims to compare the therapeutic effect of intraarticular infiltration of SH and CS in pain and temporomandibular dysfunction of patients through the reduction of pain and noises of TMJ, as well as increased jaw mobility.

\section{Materials and Methods}

\section{Subject Population}

The study design was quasi-experimental, explanatory, longitudinal, and prospective. The unit of analysis was all patients diagnosed with TMD, characterized by painful symptoms, involvement and limitation of mandibular movement, and noises or clicks in the TMJ, attended by the Dentistry Service of the Hospital Nacional Guillermo Almenara Irigoyen (HNGAI) in Lima, Peru. Patients with this lesion were treated during 2018 and were previously diagnosed by the Buco Maxilo Facial Surgery specialist. The patients were intentionally selected and divided into two groups of the same size: the SH group and the CS group. Accordingly, each group was made up of 15 participants, correlatively assigned with identification codes.

\section{Inclusion Criteria}

- Subjects of both sexes, over 18 years of age and less than 75 years old.

- Temporomandibular dysfunction with pain in the TMJ and/or limitation of mandibular movement [maximum oral aperture (MOA) less than or equal to $30 \mathrm{~mm}$, lateralities or protrusion less than $4 \mathrm{~mm}$, and/or deviation of the mandibular midline to the mouth opening].

- No response to conservative treatments (splints, medication, physiotherapy) for at least 6 months.

- Diagnostic corroboration based onto clinical and imaging findings according to the Wilkes classification. ${ }^{8}$

- Residence in Metropolitan Lima with coverage by the HNGAIESSALUD Assistance Network.

\section{Exclusion Criteria}

- Pregnant or lactating women.

- Degenerative conditions such as rheumatoid arthritis.

- Fragile health.

- Extra joint pain due to muscular, nerve, or psychogenic causes.

- Inadequate oral hygiene.

- Temporomandibular joint ankylosis, severe osteoarthritis, and disk perforation.

\section{Allocation}

The patients were divided into two groups of the same size, group $\mathrm{SH}$ and group CS. The assignment was made with identification codes in a correlative order according to the order of patient care.
It was applied in the CS group: $1 \mathrm{~mL}$ of betamethasone (Diprospan) and in the SH group: $1 \mathrm{~mL}$ of SH $25 \mathrm{mg}$ (Suprahyal) in the upper compartment TMJ.

\section{Clinical Examination}

Subjects with a diagnosis of TMD completed a clinical questionnaire that included gender, duration of painful symptoms, history of joint noises, involvement of the pattern of mandibular movements, and joint pathology before infiltration. The clinical questionnaire also included a visual analog scale (VAS) with progressive values from 0 to 10 for the measurement of pain, which was voluntarily answered by the patients to report the scale of pain at the beginning of the study and at each control visit. The clinical examination included measurement of the following variables: MOA, mandibular laterotrusive movement (MLM), and maximum protrusion (MP) in millimeters, measured using a digital millimetric ruler. In addition, palpation and auscultation of the TMJ was performed to determine the manifestation of joint noises (snapping, crepitus) during opening and closing of the mouth.

\section{Clinical Measurements}

For the measurement of the MOA, MLM, and MP, the distance from the midpoint of the incisal edge of the central incisors to the midpoint of the incisal edge of the lower incisors was measured as an anatomical reference. These measurements were obtained using a digital ruler (Fig. 1). On the other hand, joint noises described by patients were corroborated with the use of a stethoscope. Patients diagnosed with TMD previously underwent imaging evaluation, and the clinical findings were corroborated by panoramic radiography, tomography, and/or magnetic resonance imaging of the TMJ in a closed and open mouth. This stomatological clinical correlation allowed the diagnosis of TMD to be established according to Wilkes stages.

\section{Infiltration Protocol}

The study drugs were stored, transported, and administered by the Pharmacy Service of the Management of Diagnosis and Treatment Support Department of the HNGAI, according to the institutional manual of good clinical practice. Intra-articular infiltrations of the TMJ were performed as follows (Fig. 2):
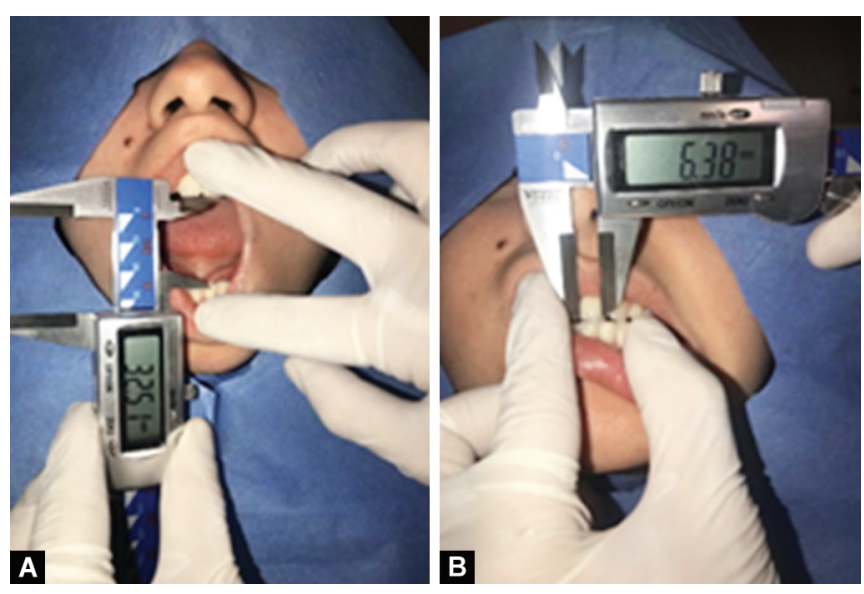

Figs 1A and B: (A) Preoperative measurement of the maximum oral opening; (B) Preoperative measurement of mandibular-laterality movement 


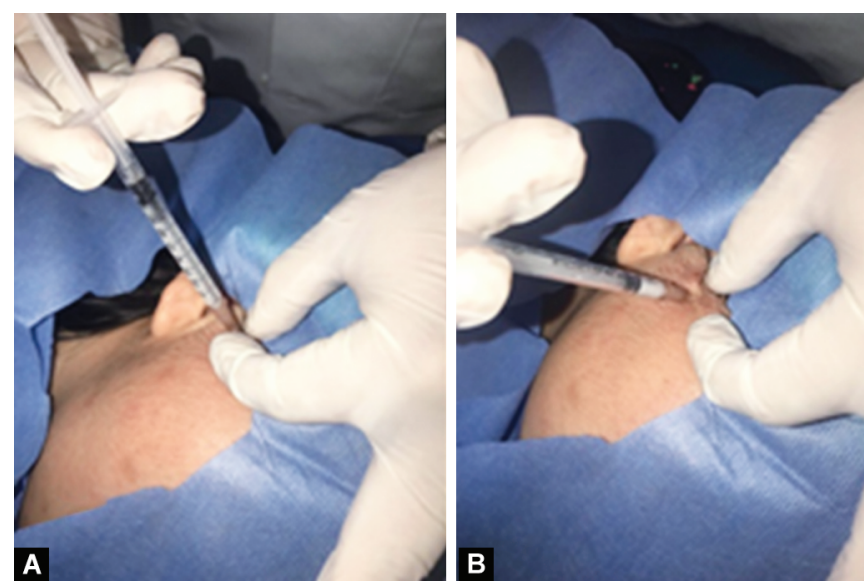

Figs $2 \mathrm{~A}$ and $\mathrm{B}$ : Registration of the intra-articular infiltration procedure: (A) Commencement of the infiltration procedure; (B) Toward the ending of the infiltration

- Anatomical knowledge of the articular and soft tissue structures to infiltrate was first obtained.

- The material required for the procedure was prepared prior to infiltration.

- The patient was placed in dorsal recumbency with the affected joint in the most comfortable and adequate position for infiltration.

- The most comfortable and safe injection route was chosen, marking the entry point if necessary.

- Rigorous asepsis was carried out using sterile gloves and sterile gauze with $95 \%$ ethyl alcohol and iodopovidone.

- The drugs were loaded with a different needle than the puncture site.

- Soft tissues of potentially painful areas were previously anesthetized on very superficial planes.

- The needle was introduced in a controlled manner without unnecessary multidirectional movements or overcoming unexpected resistance to the passage of the needle.

- Prior to the injection of the medication, using gentle aspiration, ensure no blood vessel is affected.

- In the case of nerve tissue infiltration, the patient was asked about the presence of lancinating pain in order to avoid an increase in pain or neuron damage.

- A volume was administered that does not exceed that admitted for each affected joint, $1 \mathrm{~mL}$ of $\mathrm{SH}$ (group $\mathrm{SH}$ ) or $1 \mathrm{~mL}$ of CS (group CS).

- After infiltration, the needle was carefully removed and protected with a needle cap and the puncture point was dressed.

- A soft diet was recommended for 48 hours. All patients were examined by the principal investigator who performed the infiltrations, and then at the 1st and 2nd week and 1st and 2nd month the following variables were evaluated: pain and noise in TMJ, MOA, MLM, and MP.

The development of any adverse event was duly documented in the adverse events report sheet, which contained three items with respect to the event during the study of pathology or concomitant medication and medical claims.

\section{Statistical Analysis}

The processing and statistical analysis of the results was carried out by means of a descriptive analysis of the random variables (means and standard deviation). The behavior over time of the variables of each study group was evaluated by linear regression. The relationship between the two groups in relation to the dimensions of pain, MOA, MLM, and MP was studied using the Student's test for related and independent samples, when normality was previously corroborated by the Shapiro Wilk test. Similarly, in all the dimensions of the variable, the equality of variance was corroborated in advance by the Levene test. The relationship of joint noises in both study groups was analyzed using the chi-square test $\left(\chi^{2}\right)$. The statistical analyses were performed with the statistical software SPSS version 22.0 , with a value of $p<0.05$ being considered statistically significant.

\section{Ethical Considerations}

This quasi-experimental trial was registered and approved by the Institutional Committee of Research Ethics of the Institute of Tropical Medicine of the Universidad Nacional Mayor de San Marcos CIEI-2019-002.

\section{Results}

It was found that in both $\mathrm{SH}$ and $\mathrm{CS}$ groups the predominant sex was female, and that the affected TMJ was mainly on the right side with $55.5 \%$ and $44.4 \%$, respectively. The main reasons for consultation were TMJ pain and a reduction in oral opening. A total of 14 patients $(50 \%)$ reported these reasons for consultation in both groups. Of the 30 patients examined, in the SH group it was found that the predominant score was Wilkes III (66.6\%) and IV (42.8\%), while in the CS group it was only Wilkes IV (57.1\%) (Table 1).

Pain was present in all patients and in the initial phase, a mean improvement of $7.0 \pm 1.1$ was achieved by the $\mathrm{SH}$ group compared to $6.8 \pm 0.9$ in the CS group. As shown, the decrease in pain was similar in both study groups with the greatest decrease in pain being observed between surgery and the 1st week. In both groups, TMJ pain was statistically significant at all the time points evaluated (Table 2).

The MOA increased throughout the study in both groups, resulting in a mean of $23.6 \pm 7.0 \mathrm{~mm}$ for the $\mathrm{SH}$ group and $23.7 \pm$ $6.4 \mathrm{~mm}$ for the CS group in the preoperative phase. Following drug infiltration, the MOA increased at the 1st and 2nd week and the 1st and 2 nd month controls. Statistically significant differences in MOA were only found in the 1st week and 1st months in both groups. Thus, SH and CS were found to have a favorable therapeutic effect on the increase of the MOA after TMJ infiltration (Table 2).

The MLM increased considerably at the beginning, then decreased slightly and finally remained stable along. The study presented a mean of $1.8 \pm 1.3 \mathrm{~mm}$ for the SH group and $1.9 \pm 1.2 \mathrm{~mm}$ for the CS group (preoperative time). Following drug infiltration, the MLM increased at the 1st and 2nd week and the 1st and 2nd month controls. In the 1st and 2nd months, the MLM also decreased but not statistically significantly ( $p>0.05$ ).

Therefore, SH and CS had a favorable therapeutic effect on an increase in MLM after infiltration of the TMJ in the 1st and the 2nd weeks. So it could be used as part of the treatment of this pathology (Table 2).

The MP was almost similar in the preoperative (baseline) with $2.5 \pm 1.1 \mathrm{~mm}$ for the SH group and $2.40 \pm 1.0 \mathrm{~mm}$ for the CS group; however, it increased considerably to 3.4 and $3.5 \mathrm{~mm}$ for the $\mathrm{SH}$ and CS groups, respectively, at the 2 nd month of treatment $(p<0.05)$ (Table 2) 
Table 1: Sociodemographic characteristics

\begin{tabular}{|c|c|c|c|c|c|}
\hline \multirow[b]{2}{*}{ Variable } & \multirow[b]{2}{*}{ Parameter } & \multicolumn{2}{|c|}{$\mathrm{SH}$} & \multicolumn{2}{|c|}{$C S$} \\
\hline & & $n$ & $(\%)$ & $n$ & (\%) \\
\hline \multirow[t]{2}{*}{ Sex } & Male & 2 & 13.3 & 3 & 13.3 \\
\hline & Female & 13 & 86.7 & 12 & 86.7 \\
\hline \multirow[t]{3}{*}{ Affected TMJ } & Right & 10 & 55.5 & 8 & 44.4 \\
\hline & Left & 5 & 41.6 & 7 & 58.3 \\
\hline & Both & 0 & 0.0 & 0 & 0.0 \\
\hline \multirow[t]{3}{*}{ Reason for consultation } & Pain, limitation of opening, and noise & 1 & 50.0 & 1 & 50.0 \\
\hline & Pain and limitation of opening & 14 & 50.0 & 14 & 50.0 \\
\hline & Pain and noise & 9 & 45.0 & 11 & 55.0 \\
\hline \multirow[t]{6}{*}{ Preliminary TMJ treatments } & Occlusal splint & 15 & 50.0 & 15 & 50.0 \\
\hline & Physiotherapy & 15 & 50.0 & 15 & 50.0 \\
\hline & Infiltrations & 0 & 0.0 & 0 & 0.0 \\
\hline & Arthrocentesis & 0 & 0.0 & 0 & 0.0 \\
\hline & Arthroscopy & 0 & 0.0 & 0 & 0.0 \\
\hline & TMJ open surgery & 0 & 0.0 & 0 & 0.0 \\
\hline \multirow[t]{5}{*}{ Wilkes classification } & I & 0 & 0.0 & 0 & 0.0 \\
\hline & II & 3 & 50.0 & 3 & 50.0 \\
\hline & III & 6 & 66.6 & 3 & 33.3 \\
\hline & IV & 6 & 42.8 & 8 & 57.1 \\
\hline & V & 0 & 0.0 & 0 & 0.0 \\
\hline
\end{tabular}

$\mathrm{SH}$, sodium hyaluronate; $\mathrm{CS}$, corticosteroids

Table 2: Evolution of pain in the SH and CSW groups at the different time points studied

\begin{tabular}{|c|c|c|c|c|c|c|}
\hline & & Preoperative (baseline) & First week & Second week & First month & Second month \\
\hline Outcome & Group & Mean $\pm S D$ & Mean difference & Mean difference & Mean difference & Mean difference \\
\hline \multirow[t]{3}{*}{ Pain scale } & $\mathrm{SH}$ & 7.01 .1 & -3.7 & -5.1 & -5.5 & -6.0 \\
\hline & CS & $6.8 \pm 0.9$ & -3.3 & -4.8 & -5.5 & -5.6 \\
\hline & $p$ value & & 0.001 & 0.001 & 0.001 & 0.015 \\
\hline \multirow[t]{3}{*}{ MOA } & $\mathrm{SH}$ & $23.6 \pm 7.0$ & 16.3 & 15.2 & 17.3 & 18.2 \\
\hline & CS & $23.7 \pm 6.4$ & 15.5 & 15.1 & 17.2 & 17.8 \\
\hline & $p$ value & & 0.001 & 0.521 & 0.007 & 0.461 \\
\hline \multirow[t]{3}{*}{ MLM } & $\mathrm{SH}$ & $1.8 \pm 1.3$ & 4.0 & 4.6 & 4.1 & 4 \\
\hline & CS & $1.9 \pm 1.2$ & 3.3 & 3.3 & 4 & 4.6 \\
\hline & $p$ value & & 0.001 & 0.001 & 0.014 & 0.334 \\
\hline \multirow[t]{3}{*}{ MP } & $\mathrm{SH}$ & $2.5 \pm 1.1$ & 3.0 & 3.3 & 3.7 & 3.4 \\
\hline & CS & $2.4 \pm 1.0$ & 3.0 & 3.4 & 3.8 & 3.5 \\
\hline & $p$ value & & 0.001 & 0.138 & 0.019 & 0.164 \\
\hline
\end{tabular}

$\mathrm{SH}$, sodium hyaluronate; $\mathrm{CS}$, corticosteroids; MOA, maximum oral aperture; MLM, mandibular laterotrusive movement; MP, maximum protrusion; $\mathrm{SD}$, standard deviation

Student's $t$-test; Significance level $p<0.05$.

The values were expressed in $\mathrm{mm}$ except the variable pain scale that was measured in points according to the VAS scale

According to the results obtained, it can be inferred that when comparing the therapeutic effect of intra-articular infiltration of $\mathrm{SH}$ and CS in pain and TMD of patients through the reduction of pain and noises of TMJ, as well as increased jaw mobility, both medications were effective in treating TMD in a Peruvian population.

\section{Discussion}

Temporomandibular dysfunction is a heterogeneous group of pathologies that cause pain, including joint pain, pain in the masticatory area, and headache radiating to the neck. Likewise, it limits the movement of the jaw and can induce noises and clicks in the opening or closing of the mandible. ${ }^{9-11}$ Initial treatments include conservative approaches such as local applications of heat, oral NSAIDs use, correction of occlusal abnormalities, cognitive therapy, and use of occlusal splint. These treatments are often refractory to standard TMD treatments, and therefore, intra-articular application of certain anti-inflammatory agents and substances with rheological properties in the TMJ compartment is a widely used practice, which has clearly shown to relieve pain to varying degrees and improve joint mobility. ${ }^{6,8,11}$ 
Several authors have evaluated the short-term and long-term effects of intra-articular injections of $\mathrm{SH}, \mathrm{CS}$, and saline in patients with TMD with symptomatology of the TMJ. ${ }^{7-9}$ They evaluated the effect of these different agents on subjective symptoms, clinical signs, and bite force and found that both SH and CS significantly reduced symptoms and signs, although no statistically significant differences were found in the effect between drugs in this regard. Despite this, these authors considered $\mathrm{SH}$ to be the best alternative because of its reduced risk of side effects compared to CS.

The results were similar in the present study, with both drugs reducing pain and improving mandibular mobility. However, no statistically significant differences were found between the therapeutic effect of the two drugs, with the exception of the right laterotrusion in the 1st preoperative week and at weeks 1 and 2 , which showed a better therapeutic effect with $\mathrm{SH}$. The result coincides with those of previous studies, ${ }^{12-15}$ which demonstrated the efficacy of $\mathrm{SH}$ in the reduction of pain and noise of the TMJ and an increase of mandibular mobility, simple post-infiltration, or simple arthrogenic mandibular mobility observed by arthroscopy post-infiltration. Although in the present investigation infiltration with $\mathrm{SH}$ was not accompanied by other procedures (arthrocentesis and/or arthroscopy) and the follow-up period did not exceed 2 months, $\mathrm{SH}$ proved to be effective in reducing the painful symptomatology and improving mandibular mobility. In this regard, the lubricating role of exogenous $\mathrm{SH}$ is able to reduce the action of inflammatory mediators, providing lubricating activity that remains over time, thereby reducing wear and promoting nutrition of the articular cartilage. However, the results of a study by BjØrnland et al. ${ }^{16}$ comparing the efficacy and complications of intra-articular injections of SH and CS in TMJ in 40 patients with osteoarthritis showed results that differed from those of the present study. The patients were randomly divided into two groups and received two intra-articular injections with $\mathrm{SH}$ or two intra-articular injections with CS, separated by 14 days. They concluded that SH infiltrations are more effective than CS in reducing pain and that subjects who only had joint pain without myalgic problems are the most adequate for receiving these infiltrations. On the other hand, in a study including 40 patients randomly assigned into two groups to receive intra-articular injections of SH or CS, Møystad et al. ${ }^{17}$ evaluated the results by computed tomography before and after the TMJ injections of the two drugs. Bilateral examinations of the TMJ were performed with high-resolution tomography before and 6 months after treatment, showing progression, regression, and absence of changes in bone abnormalities in TMJ, respectively. Six months after treatment, no significant differences were found between the two groups, and nor were alterations of osteoarthritic abnormalities observed in either the treated TMJ or in the contralateral joint after treatment with intra-articular injection with SH or CS. Correlatively, in the present therapeutic clinical study, no statistically significant differences were found between the therapeutic effect of drugs on pain, MOA, or MLM. Similarly, Giraddi et al. ${ }^{18}$ evaluated and compared the efficacy of CS and SH after arthrocentesis in the treatment of internal TMJ derangement in 16 patients randomized into two groups of eight receiving arthrocentesis of the upper joint space using Ringer's lactate under local anesthesia, followed by injection of betamethasone (CS) or SH. Clinical data were collected in relation to pain (VAS), MOA, joint sound, and deviation before and after up to 6 months after treatment. The results showed that the MOA improved, and a decrease in joint clicks was observed in both groups, although there were no statistically significant differences between $\mathrm{CS}$ and $\mathrm{SH}$, demonstrating that both drugs can be used with similar results. The results in the present investigation were similar, with the two drugs decreasing pain and improving mandibular mobility, although no statistically significant differences were found between the therapeutic effect of the drugs, with the exception of the right laterotrusive.

In another study, Gencer et al. ${ }^{19}$ compared the efficacy of intra-articular injections of $\mathrm{SH}, \mathrm{CS}$, and tenoxicam, and changes in subjective symptoms were compared with the VAS scale at follow-up visits in the 1st and 6th week after injection. They concluded that $\mathrm{SH}$ provided better pain relief compared to $\mathrm{CS}$ and tenoxicam. However, in the present study no statistically significant difference was found in the reduction of TMJ pain between $\mathrm{SH}$ and CS.

On the other hand, Arafat et al. ${ }^{20}$ compared the effects of ATM washing with ozonated water followed by ozone injection vs washing with Ringer's lactate followed by injection of CS and $\mathrm{SH}$. For this purpose, 27 patients with internal TMJ disorders who did not respond favorably to conservative treatment were randomly classified into three groups. The results of the treatment were evaluated biochemically by measurement of changes of the tumor necrosis factor in the synovial fluid pre and 1 week after the procedure, and joint pain was measured using the VAS scale. They concluded that arthrocentesis with ozonated water followed by ozone gas injection provides better results in the treatment of anterior disk displacement with reduction compared to $\mathrm{SH}$ and CS. However, SH proved to be statistically better than CS. In addition, Vingender et al. ${ }^{21}$ evaluated whether $\mathrm{SH}$ injection is more beneficial compared to CS in 37 joints. They also evaluated whether the efficacy of the therapy is influenced by the molecular weight of the hyaluronic acid and the protocol used. For this purpose, they applied CS only once, and SH three times in a row, once a week three consecutive times on one day. The results showed that patients treated with CS temporarily improved, but the symptoms returned, while significant improvement was observed in all the pain and mouth opening parameters with $\mathrm{SH}$. They concluded that $\mathrm{SH}$ was significantly more effective compared to CS, and that its three-time application seems to be the most effective treatment for reducing symptoms. In the present study by our group, no statistically significant differences were found between the therapeutic effect of the two drugs on pain, MOA, left laterality, and the MP, with the exception of the right laterotrusion at the different time points.

The absence of differences between the therapeutic effect on pain reduction of $\mathrm{SH}$ and $\mathrm{CS}$ on MOA, MLM, and MP in cases of TMD in our study is likely justified by the anti-inflammatory properties of both drugs. In this regard, it is known that $\mathrm{SH}$ relieves joint pain by reducing inflammatory mediator levels and that according to several studies, intra-articular application provides persistent beneficial effects due to its rheological properties in the TMJ. ${ }^{12-15}$ On the other hand, local or systemic administration of CS suppresses inflammation and pain by passing through the cell membrane and binding to CS receptors in the cytoplasm. Activated receptors inhibit gene expression for proinflammatory cytokines, enzymes, receptors, and adhesion molecules, while increasing the expression of genes encoding anti-inflammatory proteins such as interleukin-10 and the interleukin-1 receptor antagonist. Treatment of the TMJ with intra-articular CS has demonstrated to be effective in reducing pain and increasing jaw dynamics. ${ }^{19-22}$

The difference of the therapeutic effect between SH and CS with respect to the reduction of articular noises of the TMJ in cases 
of TMD is justified by the rheological properties of damping and viscoelasticity of $\mathrm{SH}$, which decrease the impact of chewing loads and reduce friction, and thus the friction of the internal structures of the TMJ. Since no significant difference was found between the therapeutic effects of SH and CS in cases of TMD in the present study, both drugs can be effectively used for pain relief and to improve mandibular mobility in patients undergoing intra-articular infiltration of TMJ. However, CSs are considered a more economic option and can be easily acquired compared to $\mathrm{SH}$, which has a high cost and requires specialized drug stores. On the contrary, an advantage of SH over CS is that its repetitive application does not cause systemic effects as in CS. Finally, the clinical application of each drug largely depends on the etiology of TMD disease, as well as the desired therapeutic effects, and also the experience of the surgeon specialized in this pathology.

The main strengths of the present study are that, in general, the diagnosis of TMD is based on anamnesic and clinical evaluation in relation to articular and/or muscle pain, noises, blocking, and alterations in the oral aperture pattern. However, these findings should be confirmed by tomographic studies of the TMJ with the mouth open and closed plus arthroscopy that establishes the static and functional position of the articular meniscus. This clinical diagnosis by imaging, and in some cases arthroscopy, requires a certain period of time, However, relief of painful symptomatology and improvement of mandibular mobility are priorities in patients with TMD and justify the use of intra-articular infiltration with anti-inflammatory drugs, analgesia, and with compounds such as $\mathrm{SH}$ and CS.

On the other hand, among the main limitations is that exogenous $\mathrm{SH}$ has demonstrated its ability to produce antiinflammatory effects, in addition to restoring joint lubrication. In vitro research suggests that $\mathrm{SH}$ promotes the endogenous synthesis of hyaluronic acid, reduces inflammation, and protects the cartilage by reducing pain by covering its receptors. The social value of the study lies in the peremptory possibility of immediate relief of painful symptomatology in TMD and improvement of mandibular mobility, with intra-articular infiltration of the TMJ with SH and CS, which are drugs that have demonstrated efficacy in recent studies in foreign populations. Depending on the severity of TMD, this type of treatment based on the infiltration of drugs in TMJ does not rule outpatients continuing with other treatments, such as arthrocentesis or lysis and joint lavage, arthroscopy, TMJ surgical correction, or total joint replacement, but it does provide pain relief and better jaw mobility, reducing joint noise.

\section{Conclusion}

In conclusion, $\mathrm{SH}$ has a therapeutic effect similar to $\mathrm{CS}$ in the reduction of TMJ pain, the increase in MOA, the increase in MLM, and the increase in MP in patients with TMJ dysfunction. In addition, $\mathrm{SH}$ has a greater therapeutic effect compared to CS in the reduction of TMJ noise at the 2nd week, the 1st month, and the 2nd month, after intra-articular infiltration.

\section{ACKnowledgments}

The authors would like to thank the Dentistry Service of the Guillermo Almenara Irigoyen National Hospital and the Universidad Nacional Mayor de San Marcos for providing the facilities for the recruitment of the patients of this research work and the Universidad Cientifica del Sur for providing the digital tools for the preparation of this manuscript.

\section{References}

1. Tuncel U. Repeated sodium hyaluronate injections following multiple arthrocenteses in the treatment of early stage reducing disc displacement of the temporomandibular joint: a preliminary report [published correction appears in J Craniomaxillofac Surg. 2013 Jul;41(5):355]. J Craniomaxillofac Surg 2012;40(8):685-689. DOI: 10.1016/j.jcms.2011.12.003.

2. Goiato MC, da Silva EV, de Medeiros RA, et al. Are intra-articular injections of hyaluronic acid effective for the treatment of temporomandibular disorders? A systematic review. Int J Oral Maxillofac Surg 2016;45(12):1531-1537. DOI: 10.1016/j. ijom.2016.06.004.

3. Moldez MA, Camones VR, Ramos GE, et al. Effectiveness of intraarticular injections of sodium hyaluronate or corticosteroids for intracapsular temporomandibular disorders: a systematic review and meta-analysis. J Oral Facial Pain Headache 2018;32(1):53-66. DOI: 10.11607/ofph.1783.

4. Yapici-Yavuz G, Şimşek-Kaya G, Oğul H. A comparison of the effects of methylprednisolone acetate, sodium hyaluronate and tenoxicam in the treatment of non-reducing disc displacement of the Temporomandibular joint. Med Oral Patol Oral Cir Bucal 2018;23(3):e351-e358. DOI: 10.4317/medoral.22237.

5. Machado E, Bonotto D, Cunali PA. Intra-articular injections with corticosteroids and sodium hyaluronate for treating temporomandibular joint disorders: a systematic review. Dental Press J Orthod 2013;18(5):128-133. DOI: 10.1590/s2176-94512013000500021.

6. Sabogal Á, Asencios J, Robles A, et al. Epidemiological profile of the pathologies of the oral cavity in a Peruvian population: a 9-year retrospective study of 18,639 patients. ScientificWorldJournal 2019;2019:2357013. DOI: 10.1155/2019/2357013.

7. Yang W, Liu W, Miao C, et al. Oral glucosamine hydrochloride combined with hyaluronate sodium intra-articular injection for temporomandibular joint osteoarthritis: a double-blind randomized controlled trial. J Oral Maxillofac Surg 2018;76(10):2066-2073. DOI: 10.1016/j.joms.2018.04.031.

8. Morey-Mas MA, Caubet-Biayna J, Varela-Sende L, et al. Sodium hyaluronate improves outcomes after arthroscopic lysis and lavage in patients with wilkes stage III and IV disease. J Oral Maxillofac Surg 2010;68(5):1069-1074. DOI: 10.1016/j.joms.2009.09.039.

9. Ferreira N, Masterson D, Lopes de Lima R, et al. Efficacy of viscosupplementation with hyaluronic acid in temporomandibular disorders: a systematic review. J Caraniomaxillofac Surg 2018;46(11):1943-1952. DOI: 10.1016/j.jcms.2018.08.007.

10. Long X. Intra-articular injections of hyaluronic acid for anterior disc displacement of temporomandibular joint. Zhonghua Kou Qiang Yi Xue Za Zhi [Internet] 2017;52(3):161-165. DOI: 10.3760/cma.j.i ssn.1002-0098.2017.03.007.

11. Fernández-Hernández S, Brenes-Ortega L, Chávarri-Prado $D$, et al. Intraarticular injections of hyaluronic acid as an alternative to corticosteroids in the treatment of osteoarthritis of the temporomandibular joint. study of systematic review. Int J Odontostomatol 2017;11(2):157-164. DOI: 10.4067/S0718381X2017000200007.

12. Alpaslan GH, Alpaslan C. Efficacy of temporomandibular joint arthrocentesis with and without injection of sodium hyaluronate in treatment of internal derangements. J Oral Maxillofac Surg [Internet] 2001;59(6):613-618. DOI: 10.1053/joms.2001.23368.

13. Sato $S$, Goto $S$, Kasahara $T$, et al. Effect of pumping with injection of sodium hyaluronate and the other factors related to outcome in patients with non-reducing disk displacement of the temporomandibular joint. Int J Oral Maxillofac Surg 2001;30(3): 194-198. DOI: 10.1054/ijom.2000.0049.

14. Sato $\mathrm{S}$, Ohta $\mathrm{M}, \mathrm{Ohki} \mathrm{H}$, et al. Effect of lavage with injection of sodium hyaluronate for patients with nonreducing disk displacement of the temporomandibular joint. Oral Surg Oral Med Oral Pathol Oral Radiol Endod 1997;84(3):241-244. DOI: 10.1016/s1079-2104(97) 90337-1. 
15. Bertolami CN, Gay T, Clark GT, et al. Use of sodium hyaluronate in treating temporomandibular joint disorders: a randomized, double-blind, placebo-controlled clinical trial. J Oral Maxillofac Surg 1993;51(3):232-242. DOI: 10.1016/s0278-2391(10)80163-6.

16. BjØrnland T, Gj/Erum AA, MØystad A. Osteoarthritis of the temporomandibular joint: an evaluation of the effects and complications of corticosteroid injection compared with injection with sodium hyaluronate. J Oral Rehabil 2007;34(8):583-589. DOI: 10.1111/j.1365-2842.2007.01759.x.

17. Møystad A, Mork-Knutsen BB, Bjørnland T. Injection of sodium hyaluronate compared to a corticosteroid in the treatment of patients with temporomandibular joint osteoarthritis: a CT evaluation. Oral Surg, Oral Med Oral Pathol Oral Radiol Endodontol 2008;105(2): e53-e60. DOI: 10.1016/j.tripleo.2007.08.024

18. Giraddi GB, Siddaraju A, Kumar B, et al. Internal derangement of temporomandibular joint: an evaluation of effect of corticosteroid injection compared with injection of sodium hyaluronate after arthrocentesis. J Maxillofac Oral Surg 2012;11(3):258-263. DOI: 10.1007/s12663-011-0324-8.
19. Gencer ZK, Özkiriş M, Okur A, et al. A comparative study on the impact of intra-articular injections of hyaluronic acid, tenoxicam and betametazon on the relief of temporomandibular joint disorder complaints. J Cranio-Maxillofacial Surg 2014;42(7):1117-1121. DOI: 10.1016/j.jcms.2014.01.041.

20. Arafat $S$, Chehata I. Clinical and biochemical assessment of different injection materials following arthrocentesis for the treatment of internal derangement of the temporomandibular joint: a comparative study. Tanta Dent J 2016;13(2):102. DOI: 10.4103/1687-8574. 188909.

21. Vingender S, László R, Benedek CK, et al. Intra-articular steroid and hyaluronic acid treatment of internal derangement of the temporomandibular joint. Orv Hetil 2018;159(36):1475-1482. DOI: 10.1556/650.2018.31138.

22. Kopp S, Akerman S, Nilner M. Short-term effects of intra-articular sodium hyaluronate, glucocorticoid, and saline injections on rheumatoid arthritis of the temporomandibular joint. J Craniomandib Disord 1991;5(4):231-238. 